\title{
Multiple-Event Catastrophe Bond Pricing Based on CIR-Copula-POT Model
}

\author{
Wen Chao $\mathbb{i D}^{1,2}$ and Huiwen Zou ${ }^{1,2}$ \\ ${ }^{1}$ School of Economics and Management, Fuzhou University, Fuzhou 350116, China \\ ${ }^{2}$ Institute of Investment and Risk Management, Fuzhou University, Fuzhou 350116, China \\ Correspondence should be addressed to Wen Chao; chaowen2014@163.com
}

Received 19 December 2017; Accepted 5 April 2018; Published 25 June 2018

Academic Editor: Alicia Cordero

Copyright ( $\odot 2018$ Wen Chao and Huiwen Zou. This is an open access article distributed under the Creative Commons Attribution License, which permits unrestricted use, distribution, and reproduction in any medium, provided the original work is properly cited.

Catastrophe events are attracting increased attention because of their devastating consequences. Aimed at the nonlinear dependency and tail characteristics of different triggered indexes of multiple-event catastrophe bonds, this paper applies Copula function and the extreme value theory to multiple-event catastrophe bond pricing. At the same time, floating coupon and principal payoff structures are adopted instead of fixed coupon and principal payoff structures, to reduce moral hazard and improve bond attractiveness. Furthermore, we develop a CIR-Copula-POT bond pricing model with CIR stochastic rate and estimate flood multiple-event triggered catastrophe bond price using Monte Carlo simulation method. Finally, we implement the sensitivity analysis to show how catastrophe intensity, maturity date, and the dependence affect the prices of catastrophe bonds.

\section{Introduction}

Different kinds of natural disasters occur frequently in the world over the past several years. These low-frequency and high-losses catastrophic events have a serious influence on peoples life and the stability society. Traditionally, when the catastrophic events occur, the national finance and social aid would be used to compensate the catastrophe losses. When faced with the natural disaster losses, the insurance companies themselves cannot satisfy the demand of catastrophe risk due to the large financial pressure and the restrictions of business ability. In recent years, there appear some kinds of important insurance-linked securities (ILSs) in the international catastrophe insurance market. And catastrophe (CAT) bonds are one of the most prominent ILSs, which transfer the consequence of CAT financial risks from issuers to investors. CAT bonds not only improve the risk bearing capacity of insurance companies, but also bring more investment choices to capital market. Reasonable pricing is the critical point in the CAT bonds issuing and trading.

Recently, some research efforts have been devoted to the catastrophe bonds pricing. Lee and Yu [1] developed a contingent-claim model to price CAT bonds with consideration of default risk, basis risk, and moral hazard. Egami and Young [2] presented a method for pricing structured CAT bonds based on utility indifference pricing. Härdle and Cabrera [3] calibrated the government parameter indextriggered CAT bonds with Mexico earthquake data; they proved that mixing reinsurance with CAT bond can reduce exposure risk and default risk. Z. G. Ma and C. Q. Ma [4] proposed a mixed approximation method to find the numerical solution for the price of catastrophe risk bonds. Nowak and Romaniuk [5] implemented the CAT bond pricing model described by the two-factor Vasicek model. Moreover, they proposed an automated approach for decision-making in fuzzy environment with relevant examples presenting this method.

While the catastrophe risks have obvious thick tail features, it is more reasonable to use extreme value theory (EVT) to characterize the tail characteristic of catastrophic losses distribution. Zimbids et al. [6] studied the Greece earthquakes data using advanced techniques from the extreme value theory. Moreover, they evaluated the CAT bond price using Monte Carlo simulation techniques and stochastic iterative equations. Shao et al. [7] applied equilibrium pricing theory and EVT to construct a multiple-variable CAT bond 
for California earthquakes. Ma et al. [8] employed a doubly stochastic Poisson and Peak over Threshold (POT) to price zero-coupon catastrophe bonds.

Since one-triggering-event CAT bond is difficult to meet the diverse needs of investors, the multiple-event CAT bond starts to rise because of its advantages. Recently, several studies have mainly focused on multiple-event CAT bonds. For example, Woo [9] addressed multiple-event risk securitization as a good way of transferring terrorism risk to capital markets. Reshetar [10] developed a framework for pricing of a multiple-event coupon paying CAT bond. It should be noted that they do not take fully the fat tail features of catastrophic risks into account. Considering that, this paper tries to combine Copula function and EVT to model a multiple-event CAT bond. The details are evaluating the marginal distributions of flood catastrophe losses and deaths via EVT. Furthermore, this paper employs the Copula function to model a joint distribution of losses and deaths. To make the price have a more applied value, we describe the spot interest rate by the CIR stochastic interest model.

The remainder of the paper is organized as follows: Section 2 briefly describes the framework of CAT claim model and stochastic interest model. Section 3 presents an empirical analysis. Section 4 is devoted to Monte Carlo simulation and sensitivity analysis. Finally, Section 5 offers conclusions for this research.

\section{Valuation Framework}

2.1. Pricing Model for the CAT Bond. Throughout this paper, we use the following assumptions:

(i) $\{N(t), t \geq 0\}$ is a Poisson process with the intensity $\lambda>0$;

(ii) $\left\{X_{i}, i=1,2, \ldots\right\}$ and $\left\{Y_{i}, i=1,2, \ldots\right\}$ denote the economy losses and the number of deaths, respectively; (iii) $\left\{X_{i}, i=1,2, \ldots\right\}$ and $\left\{Y_{i}, i=1,2, \ldots\right\}$ are two sequences of independent and identically distribution random variables;

(iv) $\{N(t), t \geq 0\},\left\{X_{i}, i=1,2, \ldots\right\}$ and $\left\{Y_{i}, i=1,2, \ldots\right\}$ are mutually independent.

We consider a coupon paying CAT bond, namely, paying a certain percentage coupons to investors at the end of the year and returning a certain percentage of principal at maturity date. In this case of catastrophe event, both coupon and principal are at risk. We choose the catastrophe losses and death tolls as trigger indicators. When one of the indicators is triggered, the current and future coupons are paid in proportion to cumulative catastrophe losses. And the principal is also paid in proportion to the cumulative losses only when both indicators are triggered simultaneously. The structures of payoff are given by the following:

the coupon paying framework:

$C_{t}$

$= \begin{cases}\prod_{i=1}^{N(t)}\left(1-\frac{\left(X_{i}-I_{X}\right)_{+}}{X_{i}}\right)\left(1-\frac{\left(Y_{i}-I_{Y}\right)_{+}}{\max \left\{Y_{i}, 1\right\}}\right) C, & \text { if } N(t)>0 ; \\ C, & \text { if } N(t)=0 ;\end{cases}$

the principal paying framework:

$$
D_{T}= \begin{cases}\prod_{i=1}^{\sum_{t=1}^{T} N(t)} q_{i}^{D} D, & \text { if } \sum_{t=1}^{T} N(t)>0 \\ D, & \text { if } \sum_{t=1}^{T} N(t)=0\end{cases}
$$

where

$$
q_{i}^{D} D= \begin{cases}\left(1-\frac{\left(X_{i}-I_{X}\right)_{+}}{X_{i}}\right)\left(1-\frac{\left(Y_{i}-I_{Y}\right)_{+}}{\max \left\{Y_{i}, 1\right\}}\right), & \text { if } X_{i}>I_{X}, Y_{i}>I_{Y} \\ 1, & \text { others, }\end{cases}
$$

$I_{X}$ and $I_{Y}$ stand for the attachment point for property losses and deaths, respectively, $C$ is coupon value, $T$ is bond maturity date, and $q_{i}^{D}$ denotes the percentage of principal paying under different cases. Moreover, in order to avoid the denominator meaninglessness (the number of deaths equal to zero), we replace $Y_{i}$ with $\max \left\{Y_{i}, 1\right\}$ in (1).

Compared with the previous researches about multipleevent CAT bond, we adopt floating coupon and principal payment structures to replace the fixed coupon and principal payment structures. This will prevent insurance companies from increasing catastrophe losses on purpose after catastrophe events, resulting in moral hazard.

Let $V$ denote the price of CAT bond; then $V$ can be calculated by the following formula:

$$
V=\sum_{t=1}^{T} E\left(C_{t}\right) p(0, t)+D_{T} \cdot p(0, T)
$$

where

$$
p(0, t)=E\left[\exp \left(-\int_{0}^{t} r(s) \mathrm{d} s\right)\right]
$$

is the price at the time $t$ of a risk-free interest rate.

2.2. Interest Rate Model. Cox, Ingersoll, and Ross (CIR) [11] model can not only describe the mean-reverting characteristic of interest rate, but also guarantee nonnegative interest rate, which is just the characteristic in real interest rate 
markets. Therefore, in this paper, the spot interest rate is assumed to follow CIR model. The interest rate model can be expressed as

$$
\mathrm{d} r(t)=\alpha(\theta-r(t)) \mathrm{d} t+\beta \sqrt{r(t)} \mathrm{d} W(t),
$$

where $\alpha>0$ is the speed of mean-reverting, $\theta>0$ is an mean of interest rate in the long run, $\beta>0$ is the volatility of the interest rate, and $W(t)$ is a standard Brownian process. In the CIR model, the risk-neutral pricing of zero-coupon bond is given by following equation:

$$
\begin{aligned}
p(0, t) & =E\left[\exp \left(-\int_{0}^{t} r(s) \mathrm{d} s\right) \mid r(0)=r\right] \\
& =A(t) \exp (-B(t) r),
\end{aligned}
$$

where

$$
\begin{aligned}
A(t) & =\left(\frac{2 \gamma e^{(\alpha+\gamma) t / 2}}{(\alpha+\gamma)\left(e^{\gamma t}-1\right)}\right)^{2 \alpha \theta / \beta^{2}}, \\
B(t) & =\frac{2\left(e^{\gamma t}-1\right)}{(\alpha+\gamma)\left(e^{\gamma t}-1\right)+2 \gamma}, \\
\gamma & =\sqrt{\alpha^{2}+2 \beta^{2}} .
\end{aligned}
$$

2.3. Extreme Value Theory and Modeling. Extreme value theory provides two methods to portray the extreme value behavior of observations, namely, Block Maxima Method (BMM) model and Peak Over Threshold model. However, BMM model is only interested in the behavior of the sample maximum, which could cause vast valid data missing. To take advantage of data information, the POT model (see [12]), which considers all observations exceeding a certain threshold value, shall be introduced to discuss the data behavior.

Suppose a random variable $X$ with the distribution function $F(x)$ and a high threshold $u ; Y=X-u$ can be viewed as the statistical extremes; then the excess distribution function of $Y$ is

$$
F_{u}(y)=P(X-u \leq y \mid X>u)=\frac{F(u+y)-F(u)}{1-F(u)},
$$

$$
y \geq 0
$$

which implies that

$$
F(x)=(1-F(u)) F_{u}(y)+F(u), \quad x \geq u .
$$

According to the theorem of $\mathrm{PBdH}$ (1975), as $u$ is large enough, the excess distribution $F_{u}(y)$ in (10) can be approximated by the generalized Pareto distribution (GPD); that is,

$$
F_{u}(y) \approx G_{\xi, \sigma}(y)= \begin{cases}1-\left(1+\xi \frac{y}{\sigma}\right), & \text { if } \xi \neq 0 \\ 1-\exp \left(-\frac{y}{\sigma}\right), & \text { if } \xi=0\end{cases}
$$

where $\xi$ and $\sigma$ are, respectively, the shape parameter and the scale parameter.

Then substituting (11) into (10), we obtain

$$
\begin{aligned}
& F(x) \\
& = \begin{cases}(1-F(u))\left(1-\left(1+\xi \frac{y}{\sigma}\right)\right)+F(u), & \text { if } \xi \neq 0 ; \\
(1-F(u))\left(1-\exp \left(-\frac{y}{\sigma}\right)\right)+F(u), & \text { if } \xi=0 .\end{cases}
\end{aligned}
$$

\section{Empirical Analysis}

3.1. Description of Data. Our data consists of flood events that are recorded in Global Archive of Large Flood Event, provided by Dartmouth College since 1985. And our study mainly considers the losses value exceeding 100 thousand dollars. Thus, the total of 827 pairs of observations for losses and deaths are picked out. Directly analyzing the data, we will find that the fitting performance of data is not very well. Aimed at improving fitting accuracy, the data is adjusted to logarithm method to eliminate the magnitude difference.

Before applying EVT, heavy-tailed characteristic of flood data should be discussed. In general, there are two methods to judge the heavy-tailed behavior: numerical method and the exponential quantile-quantile (Q-Q) plot. Here the two methods shall be used for analysis. Figure 1 obviously shows the tail of exponential QQ plots appears in a convex shape. Besides, the kurtosis values are bigger than three. Therefore, the graphs strongly suggest that the hypotheses that the data follows GPD is acceptable.

3.2. POT Model for Marginal Distribution. In POT model, if the losses after logarithm excess threshold $u_{X}$, the exceeding part would be described by a generalized Pareto distribution. For the other part, the empirical function is suitable. In conclusion, the fitting distribution of the economic losses is given by

$$
\begin{aligned}
& F_{X}(x) \\
& = \begin{cases}\widehat{F}_{X}\left(u_{X}\right)+\left(1-\widehat{F}_{X}\left(u_{X}\right)\right) G_{\xi_{X}, \sigma_{X}}\left(\ln x-u_{X}\right), & \text { if } u_{X} \leq \ln x ; \\
\widehat{F}_{X}(x), & \text { if } u_{X}>\ln x\end{cases}
\end{aligned}
$$

Since the logarithm of death number cannot be taken when its value is zero, we should take two steps to fit as follows. Firstly, for the deaths number exceeding one, we can also use the above-mentioned methods to give the following condition distribution of catastrophe death:

$$
\begin{aligned}
& F_{Y}(y \mid y \geq 1) \\
& = \begin{cases}\widehat{F}_{Y}\left(u_{Y}\right)+\left(1-\widehat{F}_{Y}\left(u_{Y}\right)\right) G_{\xi_{Y}, \sigma_{Y}}\left(\ln y-u_{Y}\right), & \text { if } u_{Y} \leq \ln y ; \\
\widehat{F}_{Y}(y), & \text { if } u_{Y}>\ln y .\end{cases}
\end{aligned}
$$

Secondly, fit the data containing zero with (14). Then the unconditional distribution is defined as 


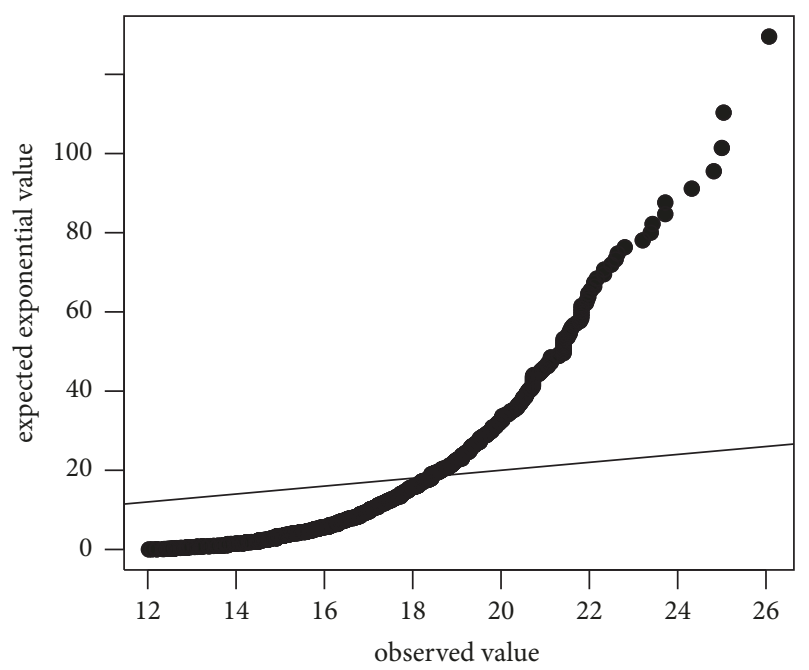

(a)

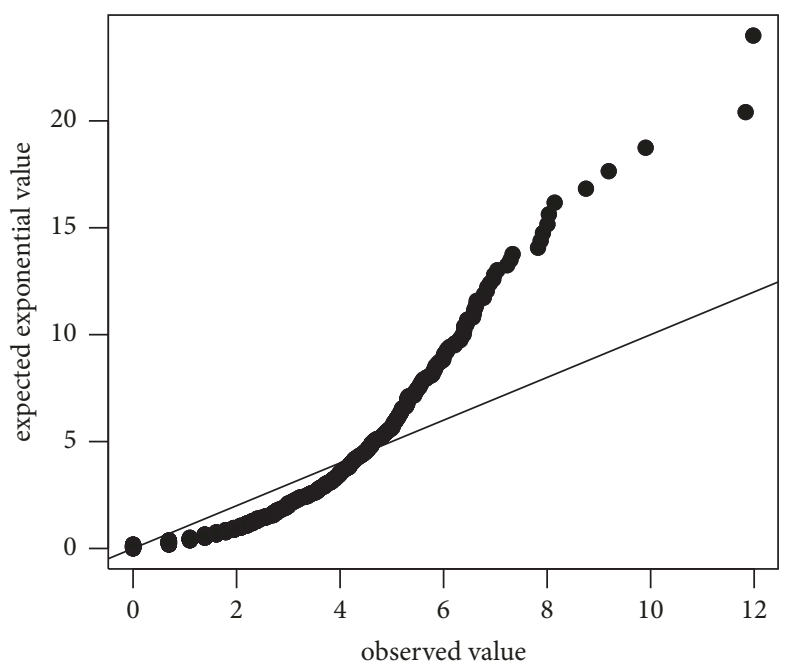

(b)

FIgURE 1: Exponential QQ plot of economic losses (a), exponential QQ plot of deaths (b).

$$
F_{Y}(y)= \begin{cases}q+(1-q)\left[\widehat{F}_{Y}\left(u_{Y}\right)+\left(1-\widehat{F}_{Y}\left(u_{Y}\right)\right) G_{\xi_{X}, \sigma_{X}}\left(\ln y-u_{Y}\right)\right], & \text { if } u_{Y} \leq \ln y ; \\ q+(1-q) \widehat{F}_{Y}(y), & \text { if } 0 \leq \ln y<u_{Y} ; \\ q, & \text { if } 0 \leq y<1 ; \\ 0, & \text { if } y<0,\end{cases}
$$

where $q$ can be evaluated with empirical distribution; namely, $\widehat{q}=N_{0} / n$, where $N_{0}$ denotes the frequency of the number of deaths equaling zero.

\subsection{Threshold Selection, Parameter Estimates, and Testing.} One of the main challenges in POT model is the selection of an optimal threshold for fitting the model. In practical application, mean excess function plot is usually used to set the threshold. Generally, the threshold is valid if the mean excess plot becomes roughly linear which starts from certain threshold level. For more details, see Embrechts et al. [13].

Figures 2 and 3 display the mean excess plots of flood losses and deaths, respectively. From Figure 2, we can see that the plot starts straight from the value 20.5 to the value 25 and seems to decline roughly after the value 25 . Based on the mean excess plot, we might choose a threshold of 20.5. Furthermore, we choose a series of threshold values and use the maximum likelihood estimation getting a series of parameters to check the validity of the threshold. If the parameter around the threshold has stability property, in this sense the selected threshold is satisfactory. Then Figure 4 indicates that the shape parameter $\xi$ and scale parameter $\sigma$ are more stable around the threshold 20.5. Similar conclusions can be drawn by considering the deaths data (Figure 5); the threshold for deaths is 5.8.
After setting the threshold values, the maximum likelihood method will be used to estimate the other parameters. Estimates are given in Table 1.

We use the diagnostic plots to check whether the GPD fits the data. Figures 6 and 7 are the diagnostic plots for losses and deaths, respectively. Almost all the dots of probability plots are on the same line. And the density plots fit well with the histograms. Consequently, it can be safely concluded that the chosen model gives a good fit for the loss and death data.

\subsection{Selection of Copula Function and Parameters Estimation.} Copula function is called joint function, which connects joint distribution function with marginal distribution function. It usually studies the nonlinear relationship among variables. And Copula function mainly includes Elliptic Copula family and Archimedean Copula family. The later was used more often because the Elliptic Copula cannot describe the asymmetric relation of variables. Therefore, in this paper, three common Archimedean Copula functions, namely, Gumbel, Clayton, and Frank Copula, will be adopted to undertake related studies.

First of all, we plug the estimates of parameters shown in Table 1 into (13) and (15). Then, via probability integral transform, the distribution series $\left\{u_{i}, v_{i}\right\}$ whose values lie in $[0,1]$ are obtained. Furthermore, it follows from Box-test that 


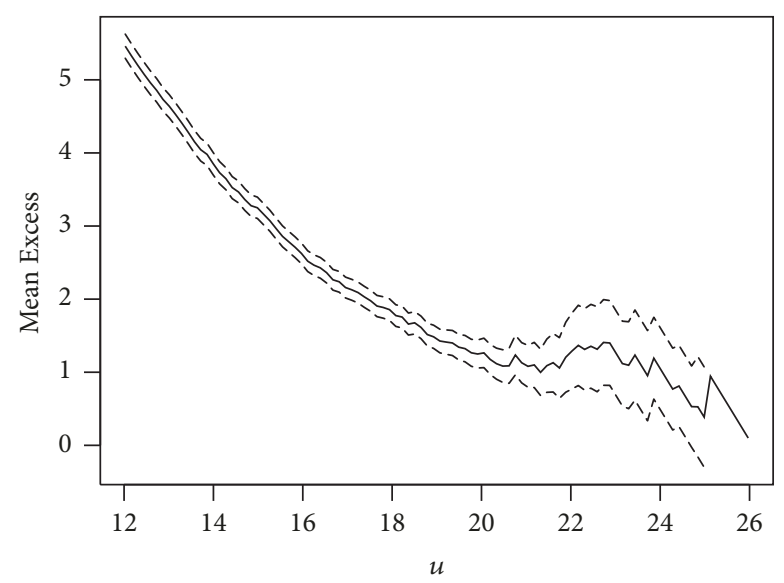

Figure 2: Mean excess plot of losses.

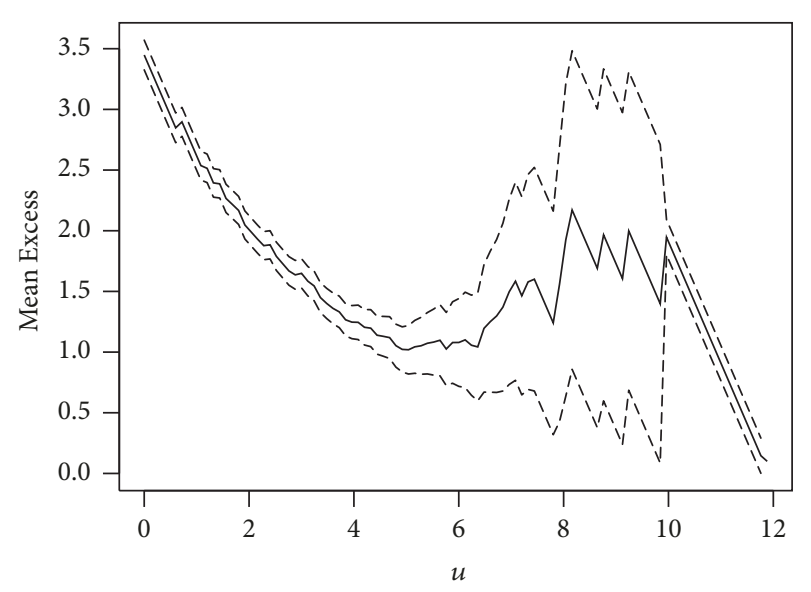

FIgURE 3: Mean excess plot of deaths.

TABLE 1: POT parameters estimates.

\begin{tabular}{lcccccc}
\hline Parameters & $\xi$ & $\sigma$ & $u$ & $N_{u}$ & $n$ & $q$ \\
\hline Economic losses & 0.0376 & 1.0428 & 20.5 & 95 & 827 & - \\
Deaths & 0.1747 & 0.8693 & 5.8 & 62 & 786 & 0.0496 \\
\hline
\end{tabular}

TABle 2: Parameter estimates of Copula function and K-S test results.

\begin{tabular}{lccc}
\hline Copula function Clayton Copula & Gumbel Copula & Frank Copula \\
\hline$\theta$ value & 0.4578 & 1.3401 & 2.3764 \\
K-S value & 0.0221 & 0.1494 & 0.5933 \\
$p$ value & 0.8292 & 0.0000 & 0.0000 \\
\hline
\end{tabular}

the series are independent. Next, let the series $\left\{u_{i}, v_{i}\right\}$ be as the observations of Copula, and use maximum likelihood method to estimate the Copula parameters. As different Copula functions reflect different dependent patterns, it is very important to choose the suitable Copula function. To attain this goal, we adopt both the Kolmogorov-Smirnov (K-S) test and Q-Q plot to test for the appropriateness of the Copula model selection. As shown in Table 2, only Clayton Copula passes the test. And from Figure 8, we can observe that the
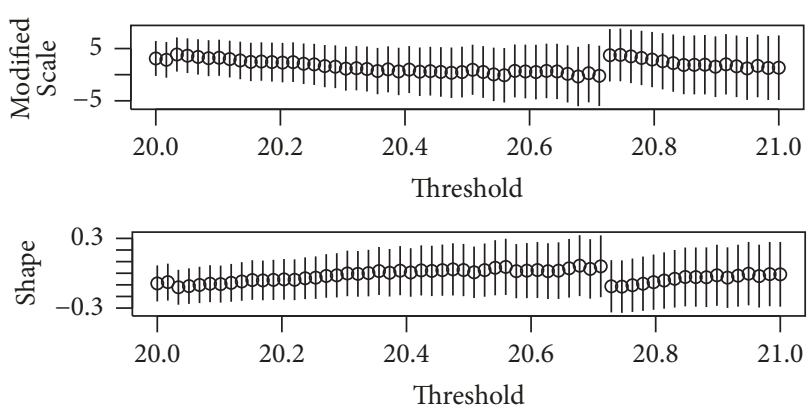

FIGURE 4: Figure of calibration scale parameters and scale parameters of losses.
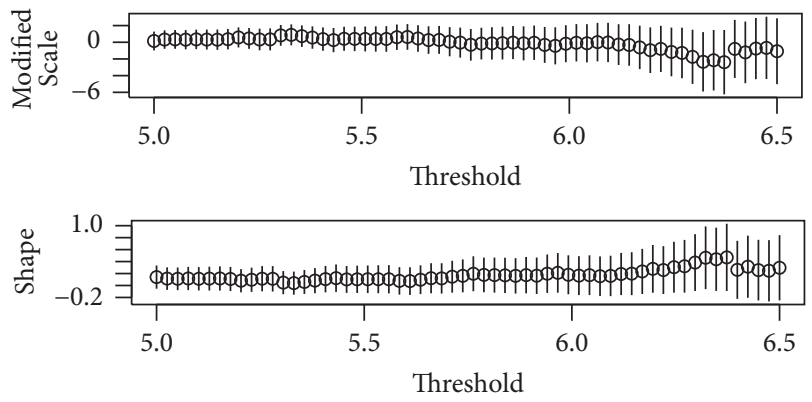

FIgURE 5: Figure of calibration scale parameters and scale parameters of deaths.

TABLE 3: Basic parameter values.

\begin{tabular}{lcc}
\hline Text interpretation & Symbol & Value \\
\hline Initial interest rate value & $r_{0}$ & 0.02 \\
Speed of mean-reverting & $\alpha$ & 0.004 \\
Long-run interest rate mean & $\beta$ & 0.02 \\
Volatility of the interest rate & $\sigma$ & 0.006 \\
Poisson process intensity & $\lambda$ & 3 \\
Face value & $F$ & $100 \mathrm{USD}$ \\
Coupon value & $C$ & 3 USD \\
Maturity time (year) & $T$ & 3 \\
Attachment point of losses & $I_{X}$ & $19.5471 \mathrm{USD}$ \\
Attachment point of deaths & $I_{Y}$ & $4.7005 \mathrm{USD}$ \\
\hline
\end{tabular}

Q-Q plot of Clayton Copula matches the straight line better than the Q-Q plots of Gumbel Copula and Frank Copula.

\section{Numerical Example and Stimulation}

4.1. Monte Carlo Simulation of CAT Bond. In this section, we will concentrate on valuing the price of CAT bond. Before the estimation, some related parameter values need to be set. According to the relative data of the real insurance market, the basic parameter values are given in Table 3.

Recalling that the explicit solutions of (1) and (2) are difficult to compute, we estimate the price of CAT bond by using Monte Carlo simulation techniques. The steps of simulation can be explicitly outlined as follows:

(1) Simulate $T$ random numbers $N(1), N(2), \ldots, N(T)$ that follow Poisson distribution with intensity $\lambda$. And these 

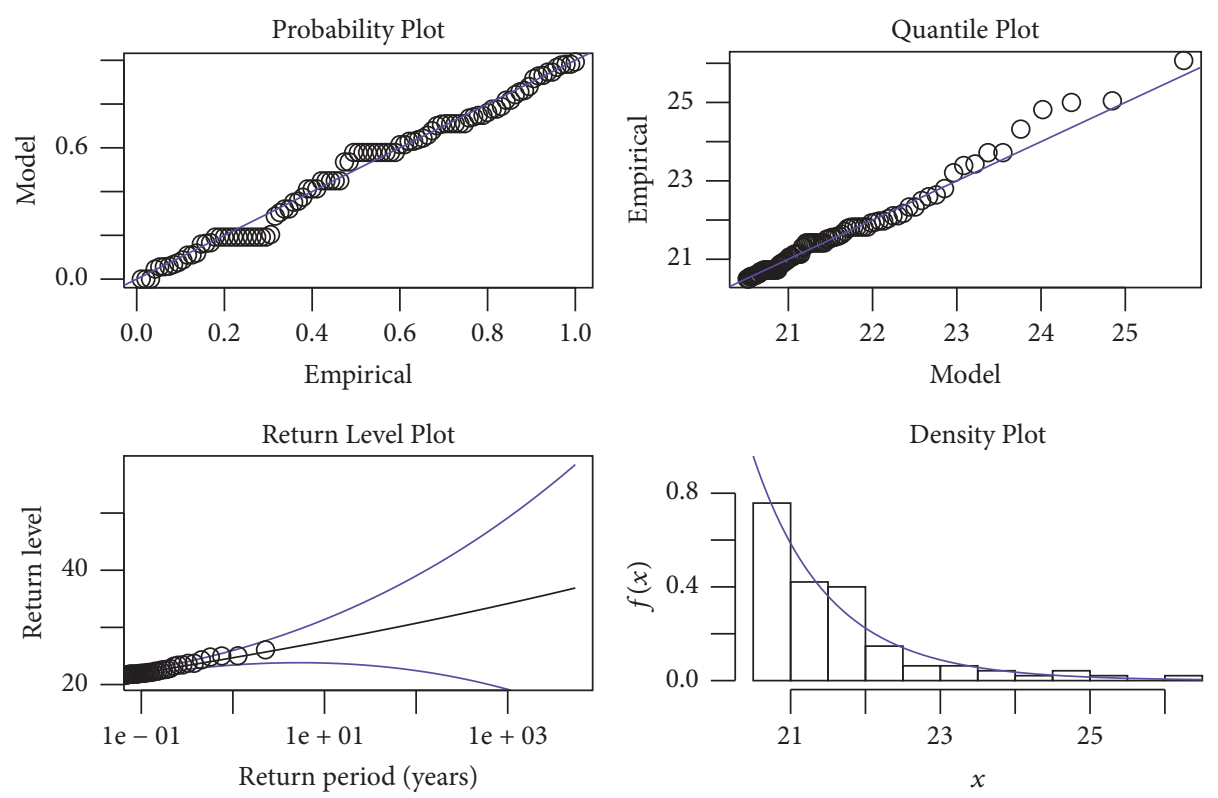

FIgURE 6: Diagnostic plots of losses.
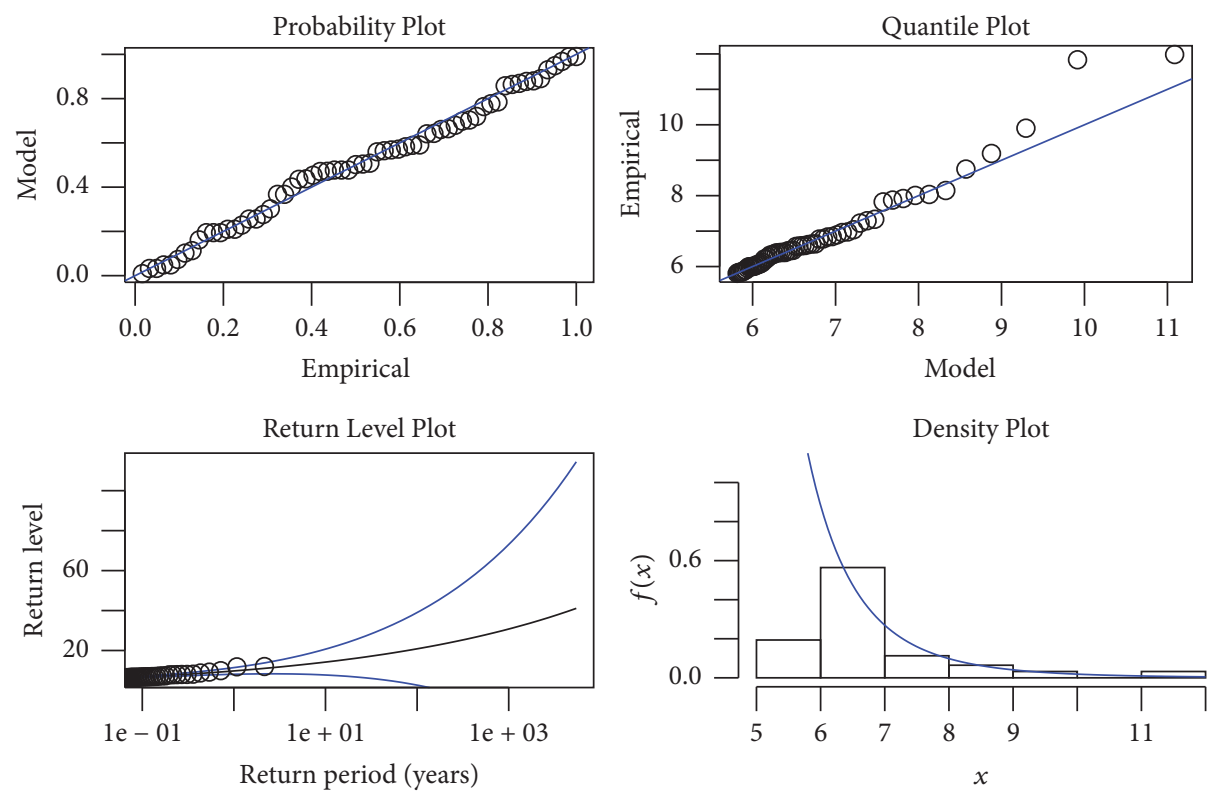

Figure 7: Diagnostic plots of deaths.

values denote the times of flood in the first year, second year, $\ldots$, and the $N$ th year, respectively.

(2) Generate $\sum_{t=1}^{T} N(t)$ pairs of random numbers $\left(u_{i}, v_{i}\right)$ from Clayton Copula function.

(3) From marginal distribution, calculate the observations about losses and deaths. They are denoted by $\left\{x_{i}, y_{i}\right\}$; that is, $x_{i}=F_{X}^{-1}\left(u_{i}\right), y_{i}=F_{Y}^{-1}\left(v_{i}\right)$.

(4) Substitute the results of procedure (3) into (1) and (2), we can get the values, and into (4), one simulation price follows.

(5) Repeat the procedures (1)-(4) for $K$ times, and calculate the mean value.
To determine the price, we implement $10^{5}$ Monte Carlo simulations and finally obtain the price of CAT bond as 57.4417 USD.

\subsection{Sensitivity Analysis for the Catastrophe Bond}

4.2.1. The Effects of Intensity $\lambda$ and Maturity Time T. The bond prices decrease as the intensity gets stronger; the discount values of coupons also show the inverse relationship with intensity from Table 4 . The main reason is that the stronger intensity means the bigger probability that catastrophe event would cause losses and deaths above the attachment points. 


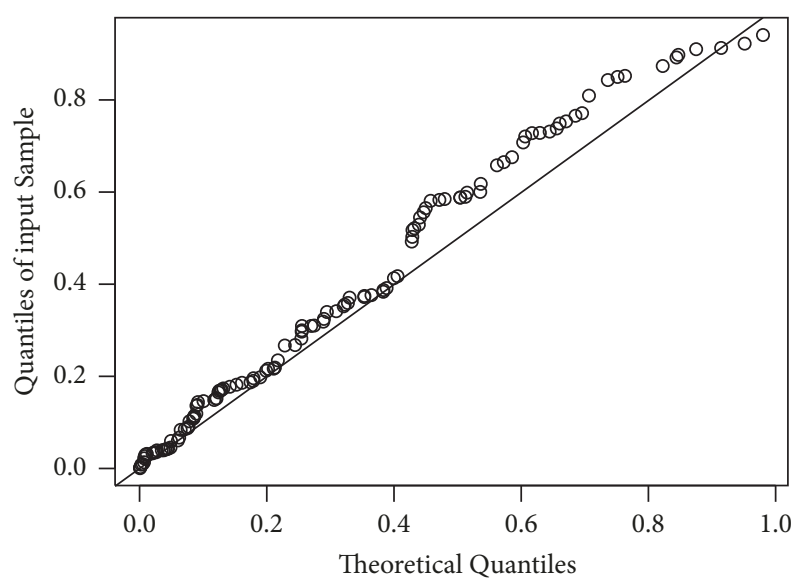

(a)

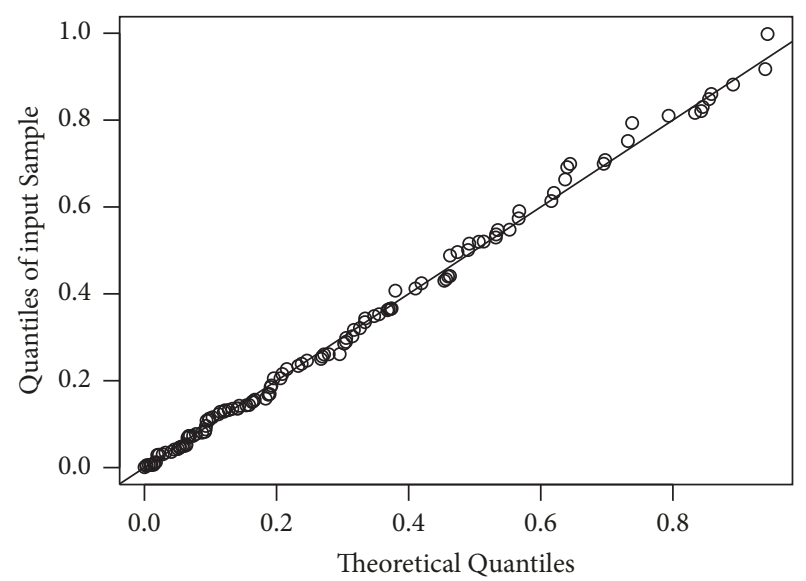

(b)

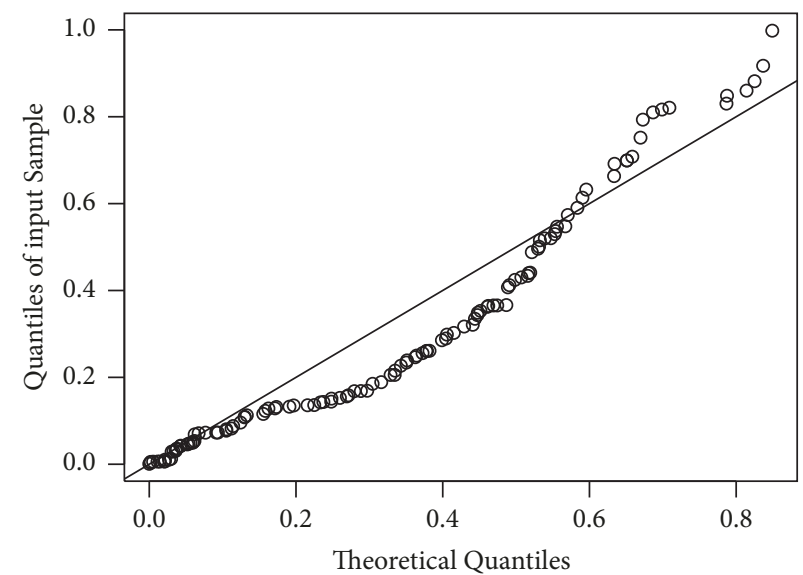

(c)

FIgure 8: Q-Q plot of Gumbel Copula (a), Clayton Copula (b), and Frank Copula (c).

TABLE 4: The sensitivity of intensity to cat bond when $T=3$.

\begin{tabular}{|c|c|c|c|c|c|}
\hline Intensity $\lambda$ & 1 & 2 & 3 & 4 & 5 \\
\hline Coupon discount & 6.8436 & 5.4078 & 4.2841 & 3.3853 & 2.6808 \\
\hline Principal discount & 77.9042 & 64.2392 & 53.1307 & 43.8916 & 36.4381 \\
\hline Bond prices & 84.3878 & 69.6470 & 57.4148 & 47.2769 & 39.1189 \\
\hline Future principal & 82.6978 & 68.1919 & 56.4000 & 46.5924 & 38.6802 \\
\hline
\end{tabular}

The result may lead the bond prices and coupon discount values to decrease.

As can be seen from Table 5, we can see that the bond prices tend to decrease with the maturity time, while the discount coupons increase with the maturity time. A possible explanation of this result may be related to the same reason with intensity effect. The effect of maturity time on bond price is totally in line with traditional bond pricing theory. Furthermore, investors can attain more coupons with the extension of maturity time; this will raise the prices of bond. Thus, the decrease of bond prices can be attributed to the results of combination of a default increase probability and an increase yielded by coupons.

As shown in Tables 4 and 5, due to the stronger intensity and the longer period of time, they both lead to a bigger probability of bonds default and, therefore, a lower price. While the two cases are different. The discount coupons increase more with the maturity time extension. However, the results of arrival intensity are totally opposite. The increase coupons can offset a part of the risk caused by the triggering bond. Thus, the impact of arrival intensity on the price of catastrophe bonds is greater than the extension of maturity time. In other words, the effect of intensity on the price of CAT bond is more pronounced than the effect of maturity time.

4.2.2. The Effect of Dependence $\theta$. As we know, the relationship between Kendall rank correlation coefficient $\tau$ and Clayton Copula parameter $\theta$ is $\tau=\theta /(\theta+2)$. The value of $\theta$ increases with $\tau$. In other words, the stronger positive 
TABLE 5: The sensitivity of maturity to cat bond when $\lambda=3$.

\begin{tabular}{lccccc}
\hline Maturity $T$ & 1 & 2 & 3 & 4 & 5 \\
\hline Coupon discount & 1.4484 & 2.8774 & 4.2791 & 5.6532 & 6.9923 \\
Principal discount & 80.7633 & 65.4306 & 53.1625 & 43.0465 & 34.8572 \\
Bond prices & 82.2117 & 68.3100 & 57.4417 & 48.6997 & 41.8495 \\
Future principal & 82.3924 & 68.0926 & 56.4337 & 46.6070 & 38.4900 \\
\hline
\end{tabular}

TABLE 6: Effect of dependence $\theta$.

\begin{tabular}{lccccc}
\hline$\theta$ & 0.0578 & 0.4578 & 0.8578 & 1.2578 & 1.6578 \\
\hline$\tau$ & 0.0281 & 0.1862 & 0.3001 & 0.3861 & 0.4532 \\
Principal discount & 0.0646 & 0.0809 & 0.0941 & 0.1056 & 0.1154 \\
Trigger frequency of two indicators triggered & 82.2117 & 68.3100 & 57.4417 & 48.6997 \\
Bond prices & 64.0219 & 57.3949 & 52.5902 & 48.5170 & 41.8495 \\
\hline
\end{tabular}

Note. The stimulation times is 100,000 .

dependence between losses and deaths implies the greater probability that the two indications are triggered at the same time. The relationship between $\theta$ and the price of bond is shown in Table 6 . The bond prices decrease as the $\theta$ increases. Therefore, when designing multiple-event CAT bonds, the less relevant indicators should be given priority; then the design goals can be achieved.

\section{Conclusions}

Catastrophe bonds triggered by multiple-event have a lower catastrophic risk than the one triggering event, while they have higher yields and more market potential than ordinary bonds. This paper designs a multiple-event triggering pricing model and combines the Copula function and the POT method in extreme value theory to study the pricing of catastrophe bonds. The pricing model not only retains the advantages of the previous multiple-event trigger model, but also has the characteristics of low risk and high return. Some improvements have been made, floating coupon and principal payment structures as replacements for the original fixed coupon and principal payment structures, so as to reduce moral hazard and improve bond attractiveness. In addition, we implement Monte Carlo simulation to price CAT bonds using Global Archive of Large Flood Event, provided by Dartmouth College since 1985. Finally, the sensitivity analysis about the parameters of pricing model is also conducted. The empirical studies reveal that the price has the inverse relationship with arrival intensity, maturity time, and Copula dependence coefficient. The effect of intensity on the price of CAT bond is more pronounced than the effect of maturity time.

In recent years, the frequency and loss affected by natural disasters in the world have been constantly expanding, which poses a more severe challenge to the traditional catastrophe insurance market. Faced with low-loss frequency and highloss severity catastrophe risk, the capacity of insurance companies for catastrophe is very limited, making catastrophe risk not yet effectively dispersed in the insurance market. Catastrophe bonds and other derivatives emerged, connecting the insurance market with the capital market and well transferring the catastrophe risk to the capital market. The model put forward in this paper enriches existing research on catastrophe bond pricing, especially in multiple-event catastrophe bond pricing. Furthermore, our research not only provides theoretical guidance for insurers to price the multiple-event catastrophe bond, but also provides a low-risk investment products to investors, enriching their investment portfolios.

\section{Conflicts of Interest}

The authors declare that they have no conflicts of interest.

\section{References}

[1] J.-P. Lee and M.-T. Yu, "Valuation of catastrophe reinsurance with catastrophe bonds," Insurance: Mathematics \& Economics, vol. 41, no. 2, pp. 264-278, 2007.

[2] M. Egami and V. R. Young, "Indifference prices of structured catastrophe (CAT) bonds," Insurance: Mathematics \& Economics, vol. 42, no. 2, pp. 771-778, 2008.

[3] W. K. Härdle and B. L. Cabrera, "Calibrating CAT bonds for Mexican earthquakes," Journal of Risk and Insurance, vol. 77, no. 3, pp. 625-650, 2010.

[4] Z. G. Ma and C. Q. Ma, "Pricing catastrophe risk bonds: a mixed approximation method," Insurance: Mathematics \& Economics, vol. 52, no. 2, pp. 243-254, 2013.

[5] P. Nowak and M. Romaniuk, "Catastrophe bond pricing for the two-factor Vasicek interest rate model with automatized fuzzy decision making," Soft Computing, vol. 21, no. 10, pp. 2575-2597, 2017.

[6] A. A. Zimbidis, N. E. Frangos, and A. A. Pantelous, "Modeling earthquake risk via extreme value theory and pricing the respective catastrophe bonds," ASTIN Bulletin, vol. 37, no. 1, pp. 163-183, 2007.

[7] J. Shao, A. Pantelous, and A. D. Papaioannou, "Catastrophe risk bonds with applications to earthquakes," European Actuarial Journal, vol. 5, no. 1, pp. 113-138, 2015.

[8] Z. G. Ma, C. Q. Ma, and S. S. Xiao, "Pricing zero-coupon catastrophe bonds using EVT with doubly stochastic Poisson arrivals," Discrete Dynamics in Nature and Society, vol. 2017, Article ID 3279647, 14 pages, 2017. 
[9] G. Woo, "A catastrophe bond niche: multiple event risk," Working Paper, NBER Insurance Group Work-Shop, Cambridge, UK, 2004.

[10] G. Reshetar, "Pricing of multiple-event coupon paying CAT bond," Working Paper, Swiss Banking Institute, 2008.

[11] J. C. Cox, J. E. Ingersoll, and S. A. Ross, "A theory of the term structure of interest rates," Econometrica, vol. 53, no. 2, pp. 385407, 1985.

[12] I. Pickands, "Statistical inference using extreme order statistics," The Annals of Statistics, vol. 3, no. 1, pp. 119-131, 1975.

[13] P. Embrechts, A. Hoing, and A. Juri, "Using copulae to bound the value-at-risk for functions of dependent risks," Finance and Stochastics, vol. 7, no. 2, pp. 145-167, 2003. 


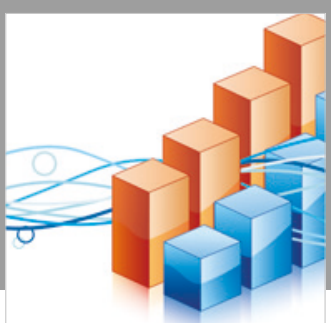

Advances in

Operations Research

\section{-n-m}
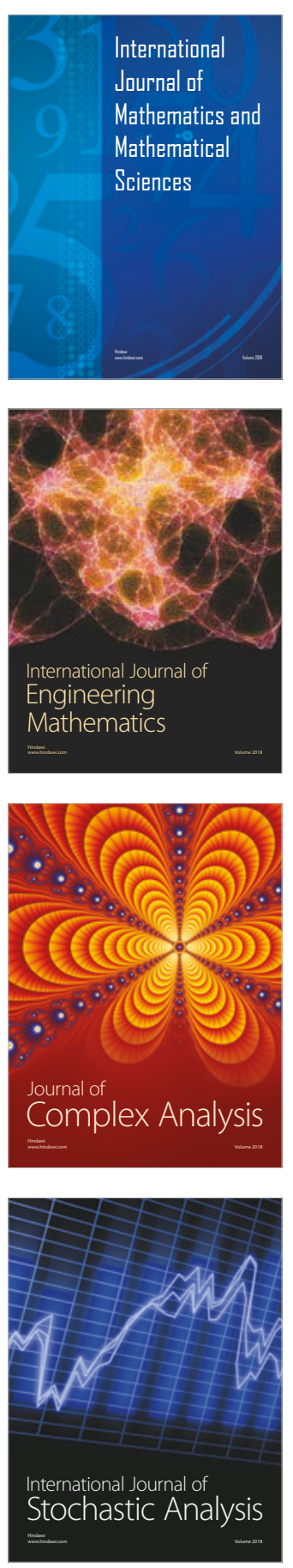
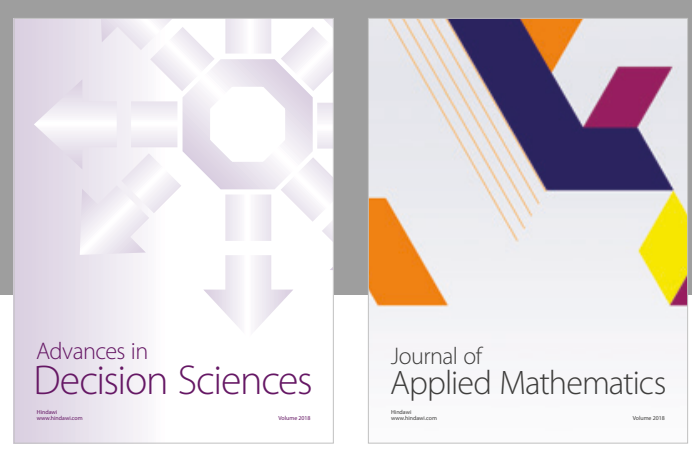

Journal of

Applied Mathematics
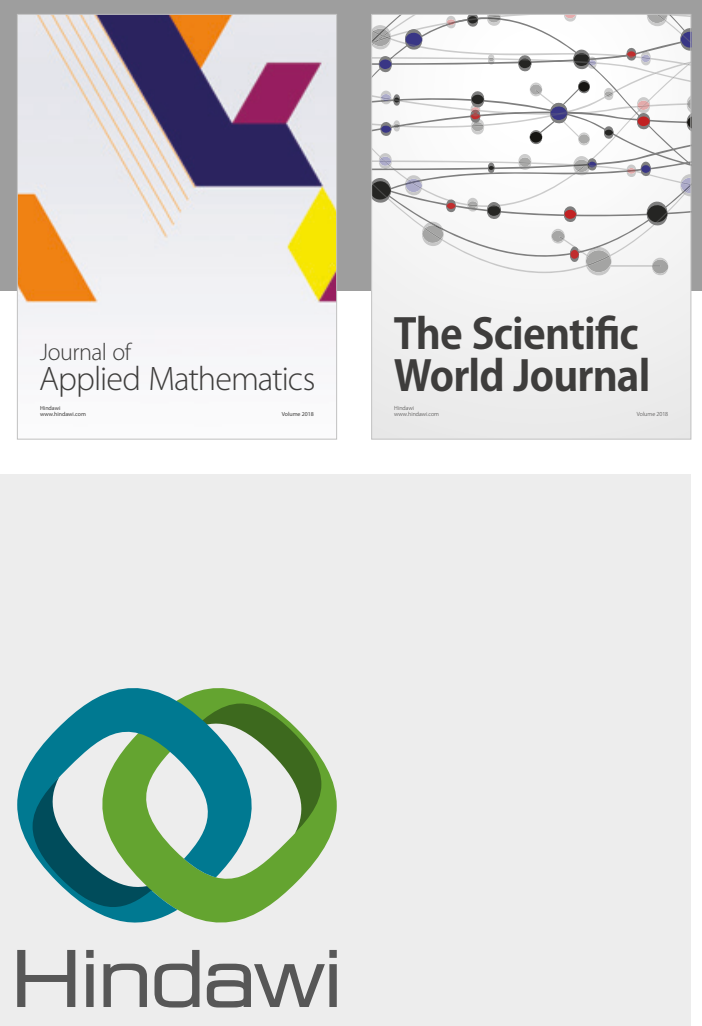

Submit your manuscripts at

www.hindawi.com

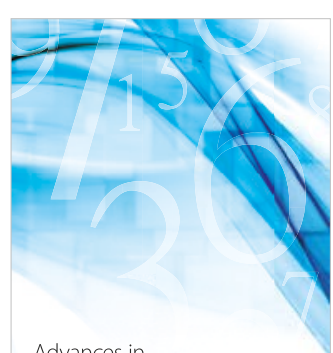

Advances in
Numerical Analysis
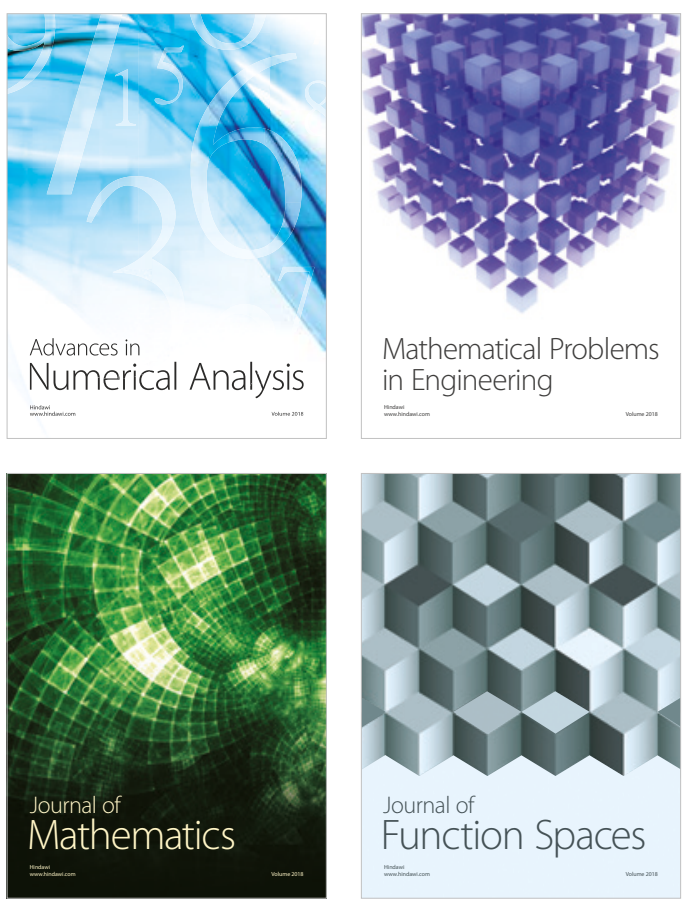

Mathematical Problems in Engineering

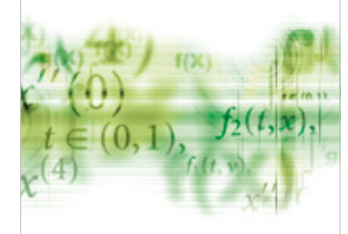

International Journal of

Differential Equations

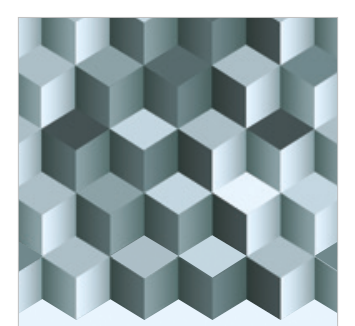

Journal of

Function Spaces

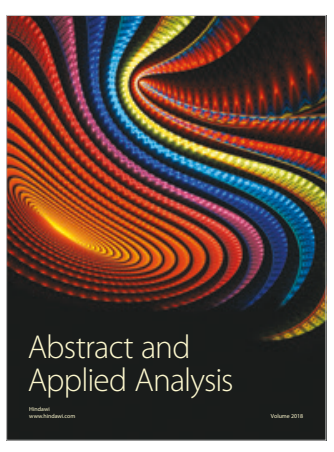

The Scientific

World Journal

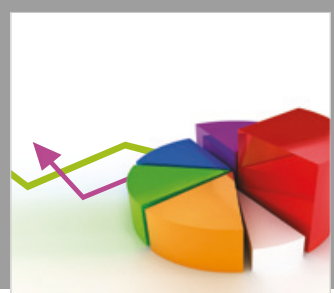

Journal of

Probability and Statistics
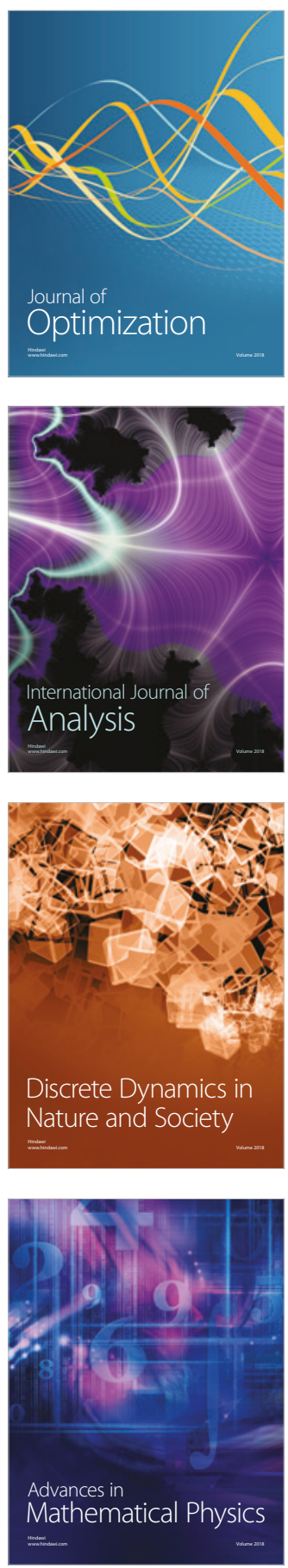